\title{
Unpaired Electrons Induced Wide-Range Light-Absorption within Zn (or Cu) MOFs Containing Electron-Withdrawing Ligands: A Theoretical and Experimental Study
}

Long Yanga ${ }^{a^{*}}$, Haijun Huang ${ }^{\mathrm{b}}$, Xuedan Luo ${ }^{\mathrm{a}}$, Huichao Hea $\mathrm{A}^{\mathrm{a}}$, Fang Gao ${ }^{\text {b* }}$, Yong Zhou ${ }^{\mathrm{c}^{*}}$

a State Key Laboratory of Environment-Friendly Energy Materials, School of Materials Science and Engineering, Southwest University of Science and Technology, Mianyang 621010, Sichuan, China. Email: yanglong@swust.edu.cn

${ }^{\mathrm{b}}$ College of Chemistry and Chemical Engineering, Chongqing University, Chongqing 400044, China. Email: fanggao1971@gmail.com

${ }^{\mathrm{c}}$ National Laboratory of Solid State Microstructures, Collaborative Innovation Center of Advanced Microstructures, Jiangsu Key Laboratory for Nano Technology, School of Physics, Nanjing University, Nanjing 210093, P. R. China. Email: zhouyong1999@nju.edu.cn

Figure S1 UV-vis diffuse-reflectance spectra of the P-DPP based Cu (a) or Zn (b)

MOFs

Figure S2 DOS spectra of P-DPP based Cu MOFs

Figure S3 EPR spectrum of P-DPP-Cu-1 $(\mathrm{g}=2.01)$

Figure S4 Dipole moment directions for Cu-based P-DPP complex compounds

Figure S5 Frontier orbitals of Zn-O-DPP compound P-DPP-Zn-2

Figure S6 Frontier orbitals of Cu-O-DPP compound P-DPP-Cu-1

Figure S7 Frontier orbitals of Cu-O-DPP compound P-DPP-Cu-2 

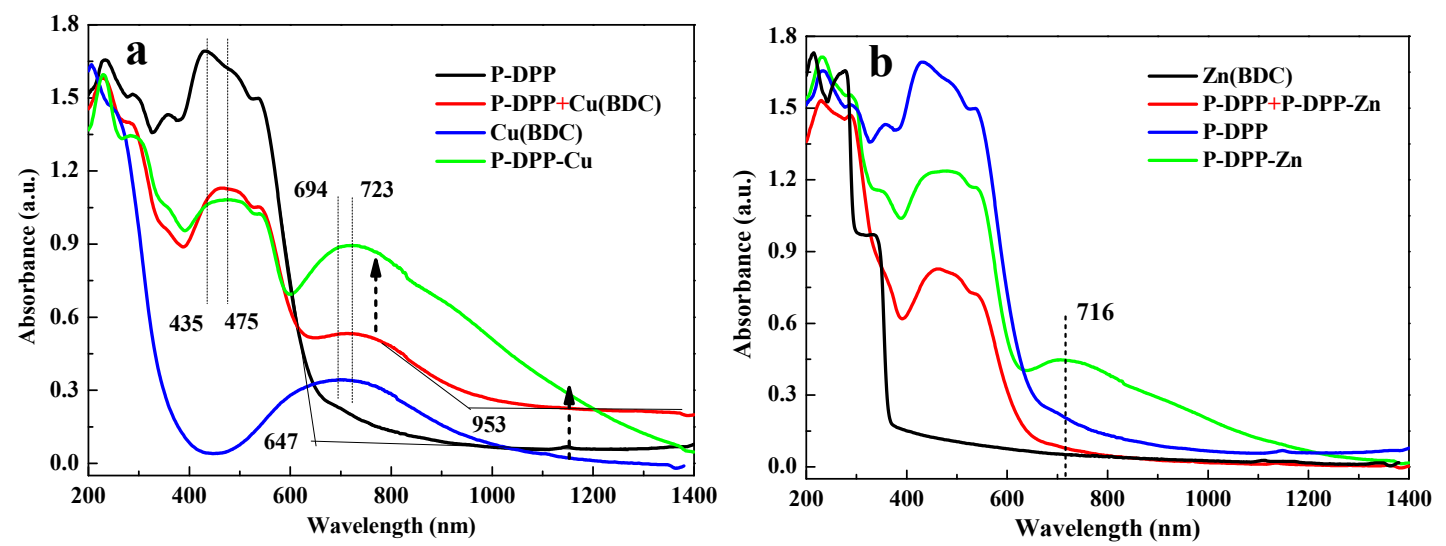

Figure S1 UV-vis diffuse-reflectance spectra of the P-DPP based Cu (a) or Zn (b) MOFs
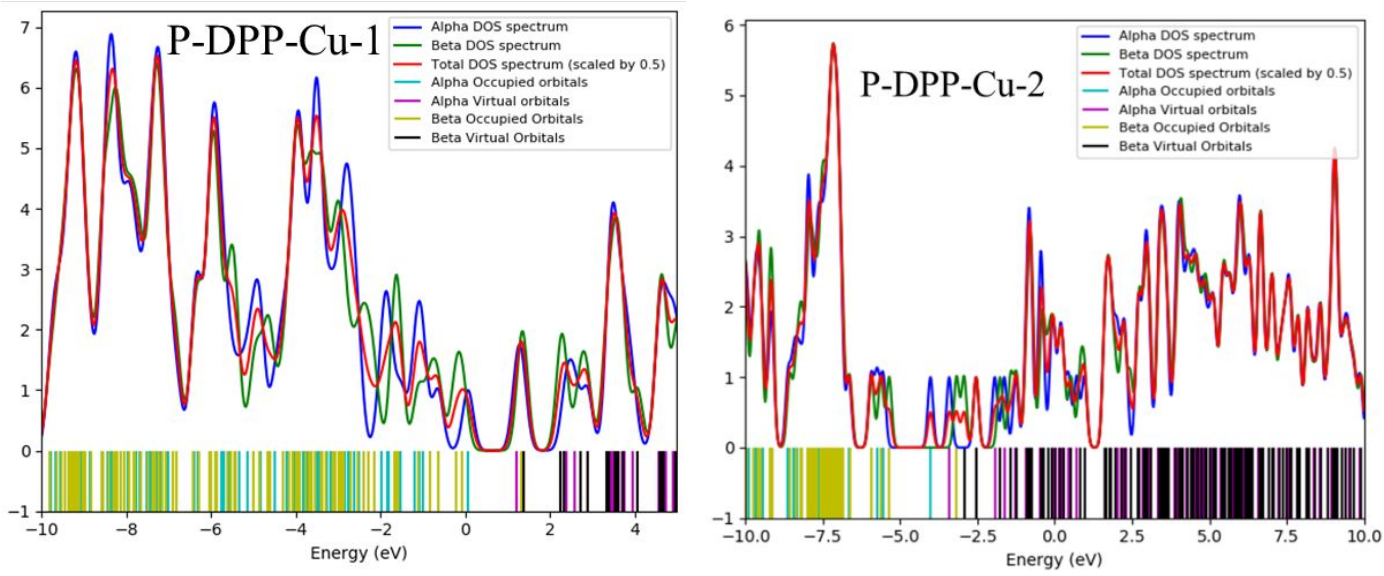

Figure S2 DOS spectra of P-DPP based Cu MOFs

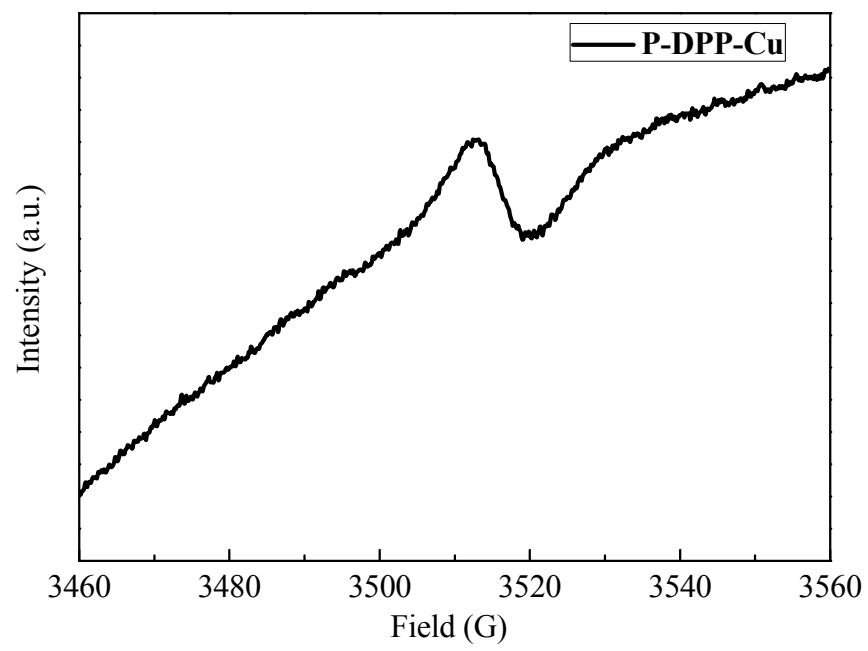

Figure S3 EPR spectrum of P-DPP-Cu $(g=2.01)$ 

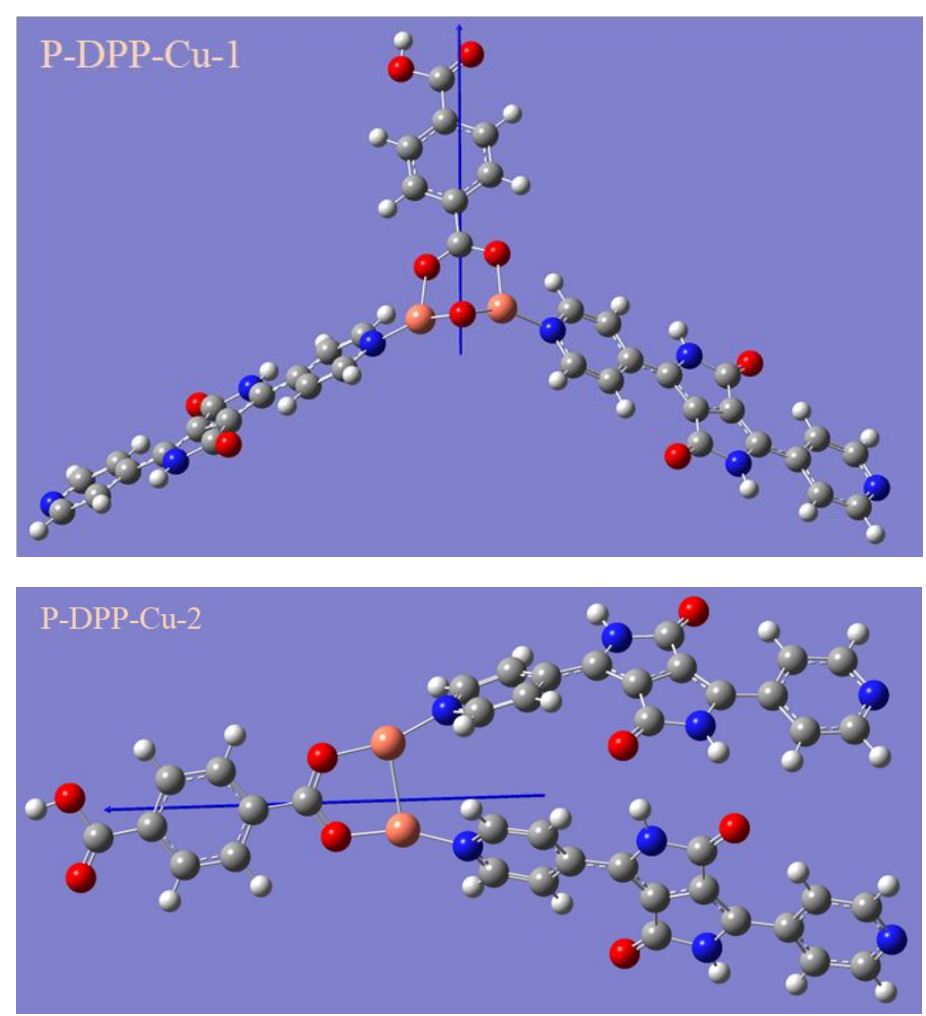

Figure S4 Dipole moment directions for Cu-based P-DPP complex compounds 

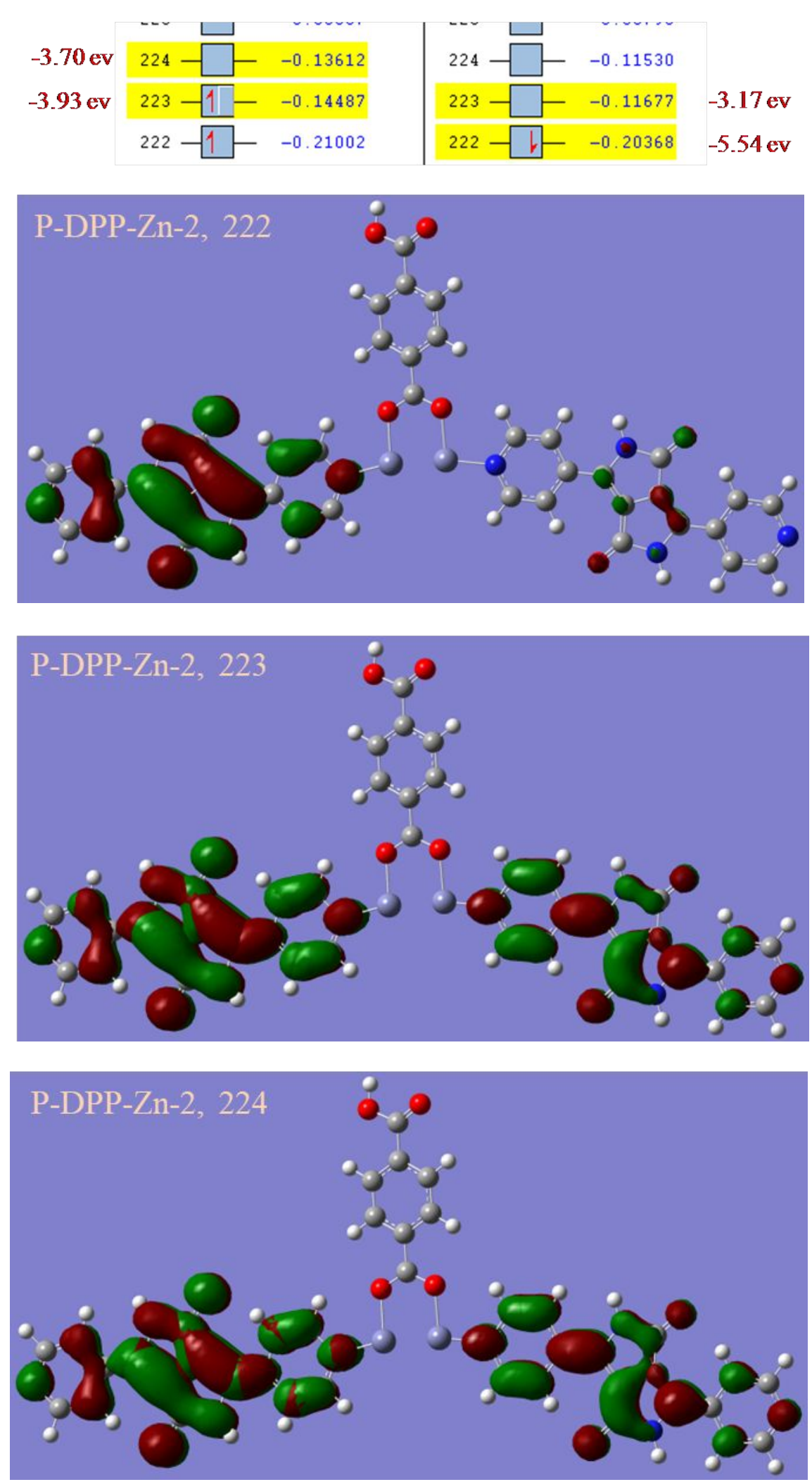

Figure S5 Frontier orbitals of Zn-O-DPP compound P-DPP-Zn-2 

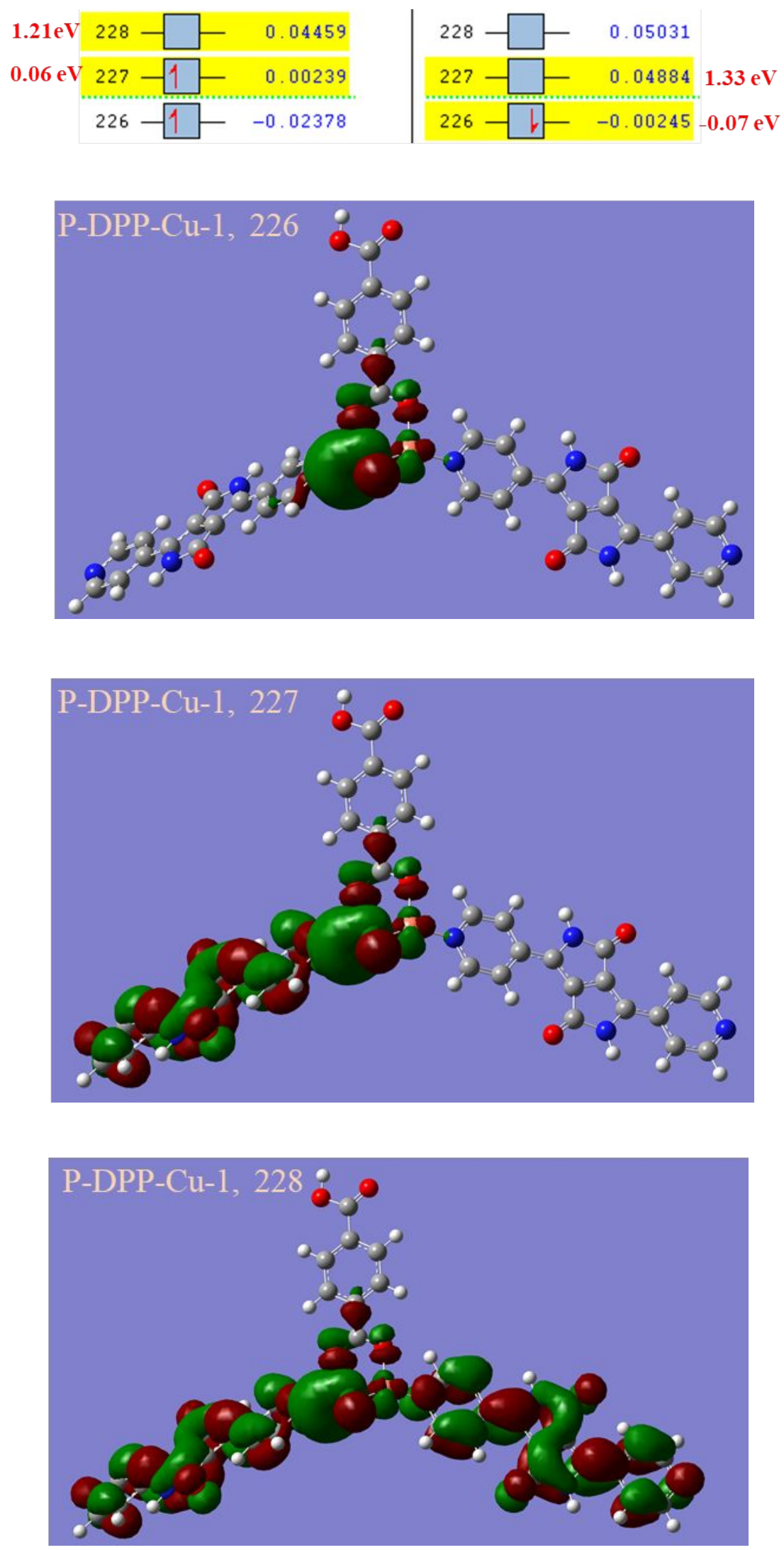

Figure S6 Frontier orbitals of Cu-O-DPP compound P-DPP-Cu-1 

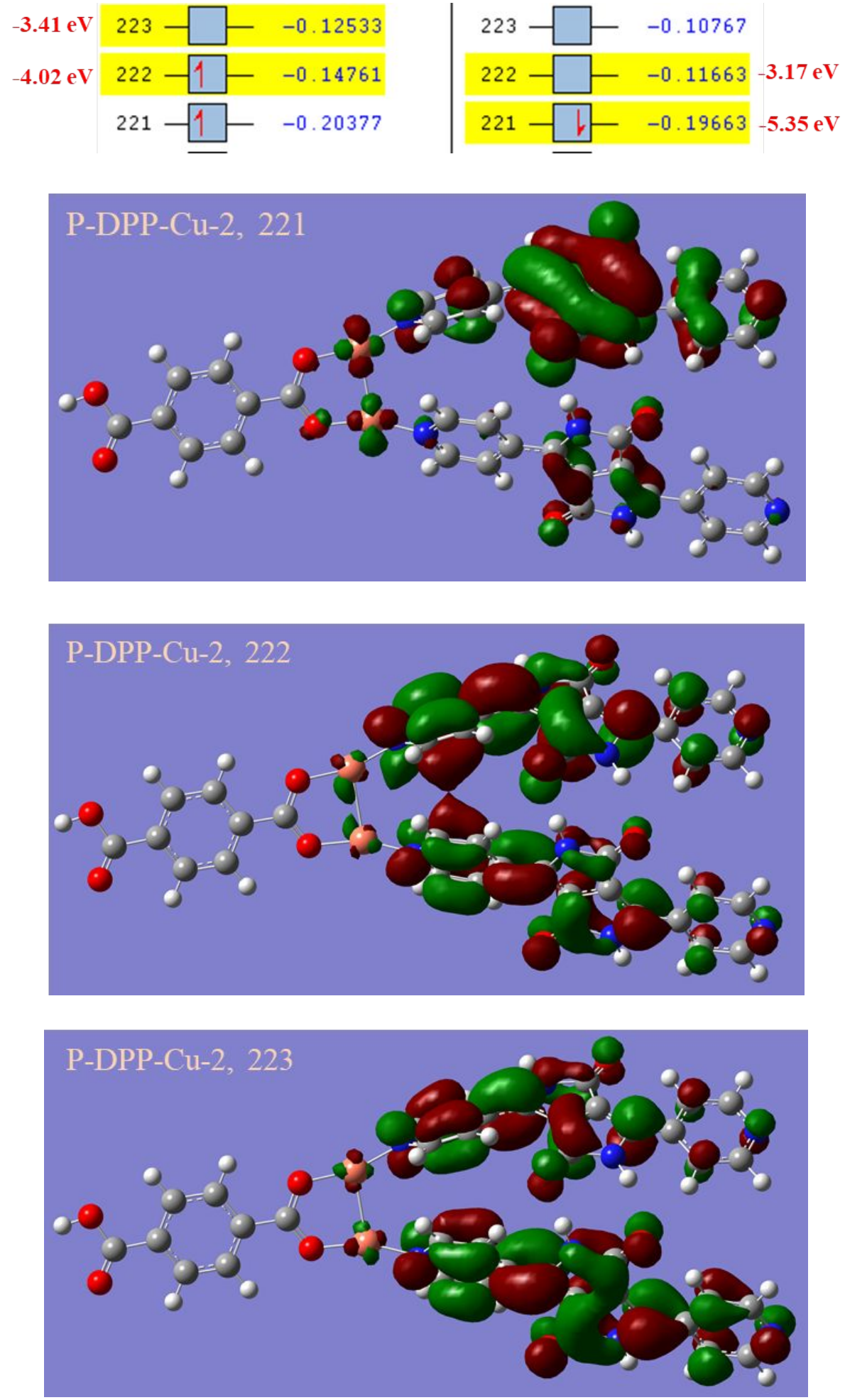

Figure S7 Frontier orbitals of Cu-O-DPP compound P-DPP-Cu-2 